\title{
Bimetric varying speed of light theories and primordial fluctuations
}

\author{
João Magueijo \\ Theoretical Physics Group, Imperial College, London, SW7 $2 B Z$
}

(Dated: October 31, 2018)

\begin{abstract}
We exhibit a varying speed of light (VSL) theory that implements the recently proposed decaying speed of sound mechanism for generating density fluctuations. We avail ourselves of bimetric VSL theories, where the speed of gravity differs from that of light. We first show that a DBI-like type of $K$-essence model has the necessary speed of sound profile to produce (near) scale-invariant fluctuations. We then examine the map between bimetric and $K$-essence models: typically the bi-scalar connecting the two metrics is a $K$-essence field in one of them. Remarkably, the DBI model is found to perturbatively represent the minimal bimetric model, where the bi-scalar is Klein-Gordon in the matter frame. But the full non-perturbative bimetric structure is even simpler: the bi-scalar dynamics should be simply driven by a cosmological constant in the matter frame, balanced by an opposite cosmological constant in the gravity frame. Thus the problem of structure formation receives an elegant and universal solution within bimetric VSL theories, which are known to also solve the flatness and entropy problems and evade a plethora of causality concerns.
\end{abstract}

PACS numbers: 0000000

\section{INTRODUCTION}

In a recent letter [1] we proposed a mechanism for producing scale-invariant density fluctuations of appropriate amplitude based on a decaying speed of sound $c_{s}$. We emphasized the mechanism's generality and how it could be implemented using a variety of methods. The examples of $K$-essence [2, 3] and varying speed of light (VSL) [4 6] were given, and other possibilities, such as non-adiabatic hydrodynamical matter, were suggested. But we stressed the generality of the proposal and deliberately chose not to marry it to any specific model. It was nevertheless noted that important issues, such as the causality concerns raised by the presence of superluminal signals or the status of the other Big Bang problems (such as the flatness or entropy problems) did require the context of a specific model. In this paper we investigate specific models, with the focus heavily trained on varying speed of light theories.

VSL theories may raise disturbing fundamental issues [7, 8, 19], at worst breaking every symmetry one can think of. But this need not be the case; indeed they may be simply regarded as non-trivial realizations of the local Lorentz group, without introducing preferred frames and other "oddities". Two implementations of this idea stand out: bimetric VSL theories and deformed special relativity. In the former the Lorentz group is realized by separate metrics for matter and for gravity, leading to different speeds for photons (and other massless "matter" particles) and gravitons [9, 10]. In the latter the dispersion relations are deformed from their usual quadratic form, so that the (group) speed of all massless particles becomes energy dependent [11]. To prevent the introduction of a preferred frame one then chooses a suitable non-linear representation of the Lorentz group [12]. In two companion papers we examine the connection between the varying speed of sound mechanism of [1] and these two types of VSL theories, concentrating on the bimetric approach here (see [14 16$]$ for connections with the latter).

Bimetric theories are perhaps the most straightforward arena for realizing the varying $c_{s}$ mechanism for producing scale-invariance. In [1] we exhibited an admittedly rather cumbersome $K$-essence theory with the required $c_{s}$ profile. Upon closer inspection, however, this model is found to be a limiting case of the Dirac-Born-Infeld (DBI) model (with the sign of one of its constants reversed with respect to what is usual, so we should perhaps call it "anti-DBI"), as we prove in this paper. Furthermore it has been shown that $K$-essence models are relieved from causality paradoxes if interpreted as bimetric models [19, 20]. In this paper we perform this exercise to discover that the DBI model universally associated with scale-invariance is precisely the minimal bimetric model, where the field linking the two metrics is provided with the simplest possible dynamics.

The plan of this paper is as follows. We first review bimetric theories that fall under the VSL remit (Section (II), stressing a couple of details of technical relevance for this paper. We then recall the varying $c_{s}$ mechanism of [1], highlighting the requirements for a viable theory of structure formation (Section III). We examine the rather wooden $K$-essence model previously proposed as "proof of concept" and show that this is in fact the limiting case of a DBI-like model (Section [V]). With this in mind we then consider the perturbative map between $K$-essence models and bimetric theories (Section $\mathrm{V}$ ), showing that the DBI model considered maps onto a simple, minimal bimetric model. A few non-perturbative features of bimetric theories are not captured by this map; but after identifying them we're able in Section VI to write down the full non-perturbative bi-metric structure behind this model, which is simply a cosmological constant with respect to the matter metric. We conclude with a discussion of other possibilities motivated by this encouraging result. 
Throughout this paper we shall use a metric with signature $(+---)$; this is for consistency with the majority of the literature quoted, but the reader is warned that some translating was required with regards to the DBI literature. We shall use the reduced Planck mass $M_{P l}=1 /(8 \pi G)$ and set the present speed of light to 1 .

\section{BIMETRIC VSL THEORIES}

Bimetric theories have been proposed as VSL theories [9, 10], solving the horizon, flatness, and dark matter problems. In the model proposed by Clayton and Moffat, for example, there are two metrics: an "Einstein" metric $g_{\mu \nu}$ (which is used to construct the Einstein-Hilbert action and defines the "Einstein" frame, also called "VSL frame" in this context); and a "matter" metric $\hat{g}_{\mu \nu}$ (used to construct the matter action via minimal coupling, and defining the "matter" or "constant $c$ " frame). The left hand side of Einstein's equations is unmodified with regards to metric $g_{\mu \nu}$; but matter is minimally coupled to $\hat{g}_{\mu \nu}$.

This set up is not unusual and has been used, for instance, in Brans-Dicke varying $G$ theories 21], where the two metrics define the Einstein and Jordan frames. What is peculiar to VSL bimetric theories is that the two metrics are related by a disformal transformation, so that the light-cones associated with them don't coincide and the speed of "light" differs from that of "gravity". TeVeS theories (an alternative to dark matter) can be seen as bimetric VSL theories [22].

The disformal transformation between the two metrics can be ruled by a choice of dynamics. In the simplest case one invokes a "bi-scalar" field $\phi$ and sets:

$$
\hat{g}_{\mu \nu}=g_{\mu \nu}+B \partial_{\mu} \phi \partial_{\nu} \phi \text {. }
$$

Here $B$ is chosen to have dimensions of $M^{-4}$, so that $\phi$ has dimensions of $M$. In the most general case $B$ could be any function of $\phi$ and $X=\frac{1}{2} \partial_{\mu} \phi \partial^{\mu} \phi$, but in the minimal theory it is a constant. The action breaks into $S=S_{g}+$ $S_{m}+S_{\phi}$ with:

$$
\begin{aligned}
S_{g} & =\frac{M_{P l}^{2}}{2} \int d^{4} x \sqrt{-g} R\left[g_{\mu \nu}\right] \\
S_{m} & =\int d^{4} x \sqrt{-\hat{g}} \mathcal{L}_{m}\left[\hat{g}_{\mu \nu}, \Phi_{M a t t}\right]
\end{aligned}
$$

and $S_{\phi}$ determining the bi-scalar dynamics. In the original formulation, due to Clayton and Moffat, $\phi$ has a Klein-Gordon Lagrangian in the Einstein $\left(g_{\mu \nu}\right)$ frame, that is

$$
S_{\phi}=\int d^{4} x \sqrt{-g}\left(\frac{1}{2} g^{\mu \nu} \partial_{\mu} \phi \partial_{\nu} \phi-V\right) .
$$

But we could also make it Klein-Gordon in the matter $\left(\hat{g}_{\mu \nu}\right)$ frame:

$$
S_{\phi}=\int d^{4} x \sqrt{-\hat{g}}\left(\frac{1}{2} \hat{g}^{\mu \nu} \partial_{\mu} \phi \partial_{\nu} \phi-V\right)
$$

or even consider hybrids, for which kinetic and potential terms live on different frames, e.g.

$$
S_{\phi}=\int d^{4} x \sqrt{-\hat{g}} \frac{1}{2} \hat{g}^{\mu \nu} \partial_{\mu} \phi \partial_{\nu} \phi-\int d^{4} x \sqrt{-g} V .
$$

The bi-scalar, after all, connects the two frames, so any of these dynamics is possible and can be considered minimal if $B$ is constant. A subtlety, to be unveiled in Section VI, reveals an even more "minimal" dynamics.

We stress two little known peculiarities of these theories, which will be of technical relevance later. First the Einstein equations remain unmodified in the Einstein frame $\left(G_{\mu \nu}=-T_{\mu \nu} / M_{P l}^{2}\right)$ only if we define the stress energy tensor as

$$
T^{\mu \nu}=\frac{2}{\sqrt{-g}} \frac{\delta}{\delta g_{\mu \nu}}\left(S_{M}+S_{\phi}\right)
$$

i.e. if we map the matter (and bi-scalar, if applicable) Lagrangian into the Einstein frame before computing $T_{\mu \nu}$. The Bianchi identities are then promptly satisfied. But if we use the matter frame definition:

$$
\hat{T}^{\mu \nu}=\frac{2}{\sqrt{-\hat{g}}} \frac{\delta}{\delta \hat{g}_{\mu \nu}}\left(S_{M}+S_{\phi}\right)
$$

(say, for theory (44)) then we have instead

$$
G^{\mu \nu}=-\frac{1}{M_{P l}^{2}} \sqrt{\frac{\hat{g}}{g}} \hat{T}^{\mu \nu}
$$

and consistency with the Bianchi identities is more intricate to prove [9]. Cosmological perturbation calculations are most easily performed in the Einstein frame, so it is important to bear this in mind.

A second peculiarity is that even though matter is minimally coupled to $\hat{g}_{\mu \nu}$ for both Lagrangian and equations of motion the same cannot be said of the bi-scalar. If the bi-scalar has a Klein-Gordon Lagrangian (either in the Einstein or matter frames) its field equations won't be Klein-Gordon, but receive a correction. This is because, upon performing variations in $S$, one finds

$$
\delta \hat{g}_{\mu \nu}=\delta g_{\mu \nu}+4 B \partial_{(\mu} \phi \partial_{\nu)} \delta \phi
$$

so that a term related to the stress-energy tensor appears in the field equation of $\phi$. If we take (3) this becomes

$$
\nabla^{2} \phi+V^{\prime}(\phi)+\sqrt{\frac{\hat{g}}{g}} B \hat{T}_{M}^{\mu \nu} \hat{\nabla}_{\mu} \hat{\nabla}_{\nu} \phi=0 .
$$

This led [9] to the introduction of a "third", derived metric, associated with the Klein-Gordon propagation of $\phi$ (see their papers for details). If we take (4) the field equation becomes:

$$
\hat{\nabla}^{2} \phi+V^{\prime}(\phi)+B \hat{T}^{\mu \nu} \hat{\nabla}_{\mu} \hat{\nabla}_{\nu} \phi=0
$$


so that the "third metric" providing the field with a Klein-Gordon type of equation:

$$
\bar{g}^{\mu \nu} \hat{\nabla}_{\mu} \hat{\nabla}_{\nu} \phi+V^{\prime}(\phi)=0
$$

is

$$
\bar{g}^{\mu \nu}=\hat{g}^{\mu \nu}+B \hat{T}^{\mu \nu} .
$$

This is not a proper space-time structure; for instance the covariant derivative $\hat{\nabla}_{\mu}$ used in (12) is still defined with respect to metric $\hat{g}_{\mu \nu}$. But it renders the field equations in a Klein-Gordon format, something that will be of use later [4].

It is a notable fact that (in the absence of matter) the Lagrangian that does produce a Klein-Gordon equation in the $\hat{g}_{\mu \nu}$ frame is a pure cosmological constant. This will be explained further in Section VI but it should already be obvious from these expressions.

\section{THE DECAYING SPEED OF SOUND MECHANISM}

We now review the mechanism for generating scaleinvariant fluctuations proposed in [1]. The central result is that for any (constant) equation of state $w=p / \rho$ scaleinvariance follows from a sound speed $c_{s} \propto \rho$, if the fluctuations originate from a vacuum state defined inside the (sound) horizon, according to the standard prescription.

Let the speed of sound diverge with conformal time according to $c_{s} \propto \eta^{-\alpha}$ (with $\alpha>0$; note that $\eta$ is positive and increases from zero). Whether we employ a hydrodynamical or a scalar field description, the density fluctuations are described by a modified harmonic oscillator equation. This can be written in terms of a variable $v$ related to the curvature perturbation $\zeta$ by $\zeta=-v / z$, with $z \propto \frac{a}{c_{s}}$. The equation for $v$ is [3, 17, 18]:

$$
v^{\prime \prime}+\left[c_{s}^{2} k^{2}-\frac{z^{\prime \prime}}{z}\right] v=0
$$

As with inflation this equation can be solved with Bessel functions, with solutions:

$$
v=\sqrt{\beta \eta}\left(A J_{\nu}\left(\beta c_{s} k \eta\right)+B J_{-\nu}\left(\beta c_{s} k \eta\right)\right) .
$$

The order $\nu$ is given by

$$
\nu=\beta\left(\alpha-\frac{3(1-w)}{2(1+3 w)}\right)
$$

and $\beta=1 /(\alpha-1)>0$. The boundary conditions, inside the (sound) horizon $\left(k c_{s} \eta \gg 1\right)$ can be found from the WKB solution:

$$
v \sim \frac{e^{i k \int c_{s} d \eta}}{\sqrt{c_{s} k}} \sim \frac{e^{-i \beta c_{s} k \eta}}{\sqrt{c_{s} k}} .
$$

These are satisfied if the integration constants $A$ and $B$ in (15) are $k$-independent numbers of order 1 . The spectrum left outside the horizon can now be found. Since $c_{s} \eta$ is a decreasing function of time, the negative order solution is the growing mode, so that asymptotically we have:

$$
v \sim \frac{\sqrt{\beta \eta}}{\left(c_{s} k \eta\right)^{\nu}}
$$

Scale-invariance of the curvature fluctuation $\left(k^{3} \zeta^{2}=\right.$ const) therefore requires $\nu=3 / 2$, i.e.

$$
\alpha=\alpha_{0}=6 \frac{1+w}{1+3 w}
$$

and if we rewrite $c_{s}$ in terms of the density $\rho$ we conclude that this implies $c_{s} \propto \rho$ for all $w$. The spectrum can also be made red or blue depending on whether $\alpha<\alpha_{0}$ or $\alpha>\alpha_{0}$, specifically

$$
n_{S}-1=\beta\left(\alpha-\alpha_{0}\right) .
$$

Finally the fluctuations' amplitude can be worked out from formula (see [3] ):

$$
k^{3} \zeta^{2} \sim \frac{(5+3 w)^{2}}{1+w} \frac{\rho}{M_{P l}^{4} c_{s}},
$$

giving further support for $c_{s} \propto \rho$ as the root of scaleinvariance. At low densities $c_{s}$ must be constant so scaleinvariance must effectively require $c_{s}=c_{0}\left(1+\rho / \rho_{\star}\right)$, where $c_{s} \approx c_{0}$ at low-energy and $\rho_{\star}$ is the density that triggers its divergence. Thus the normalization is set by the ratio of this scale to the Planck scale, viz:

$$
k^{3} \zeta^{2} \sim \frac{(5+3 w)^{2}}{1+w} \frac{\rho_{\star}}{M_{P l}^{4}} \sim 10^{-10} .
$$

For the rest of this paper we shall use bimetric VSL theories to implement this mechanism, but for the sake of clarity it is important to dissociate our efforts from those previously made 23.

In [23] a structure formation model was based on a biscalar $\phi$ which is Klein-Gordon in the Einstein frame, i.e. satisfies (3) with $V=0$. Therefore its speed of sound in the Einstein frame (where the fluctuations' calculation is done) is fixed, $c_{s}=1$. The fluctuations in $\phi$ follow Eqn. (14) with $c_{s}=1$ and decelerated expansion, and so their modes start off frozen-in (outside the horizon), oscillating only in the current epoch, when they enter the horizon. The model, thus, cannot rely on conventional methods for setting the initial conditions, i.e. by considering (vacuum quantum) fluctuations inside the (sound) horizon and then following them as they leave the horizon and freeze-out. Quite the opposite happens: the $\phi$ modes do not re-enter the horizon nowadays because they were never inside the horizon before. For the purpose of structure formation the horizon problem for $\phi$ has not been solved. Thus the need to appeal to "non-conventional" initial conditions for super-horizon modes, as considered in 13 . 
Undoubtedly the horizon problem is solved for matter in these models. But matter is subdominant and irrelevant for structure formation, as recognized in [23]. It may be that interactions between the bi-scalar and matter causally connect super-horizon modes for the bi-scalar but these interactions are assumed to be sub-dominant (indeed matter is set to zero in 23] ). The main interaction of the bi-scalar is with gravity (according to (14) with $c_{s}=1$ ), and fluctuation modes in the bi-scalar start frozen-in, not oscillating, stressing the presence of a "horizon problem".

With regards to "non-conventionality", we do not feel qualified to throw the first stone. But we do believe it unnecessary to invoke new methods for setting initial conditions to obtain scale-invariance in bimetric models, as we demonstrate in this paper.

\section{THE (ANTI-)DBI MODEL AS A REALIZATION OF SCALE-INVARIANCE}

In [1] we already exhibited a proof of concept realization of the scenario just reviewed. Here we show that this realization is the limiting case of a Dirac-Born-Infeld theory, with a crucial change in the sign of one constant with respect to what is usual in the literature.

The model in [1] is based on $K$-essence, a scalar field theory with non-trivial kinetic terms [2, 24 26]. The Lagrangian has the form

$$
\mathcal{L}=K(X)-V(\phi)
$$

(with $X=\frac{1}{2} \partial_{\mu} \phi \partial^{\mu} \phi$ ), where $K$ can be any function of $X$. Computing the stress energy tensor we find the pressure and density:

$$
\begin{aligned}
& p=K-V \\
& \rho=2 X K_{, X}-K+V
\end{aligned}
$$

whereas the speed of sound is found to be:

$$
c_{s}^{2}=\frac{K_{, X}}{K_{, X}+2 X K_{, X X}} .
$$

The cuscuton model [27, 28] is a good starting point for our construction. It is defined as a model with $K \propto$ $\sqrt{X}$. It has a number of peculiarities: its speed of sound is infinite (since the denominator of (26) vanishes); its kinetic term $K$ doesn't contribute to $\rho$ but only to $p$, so that its energy density is fully due to the potential $V$ if present. For a general potential the field can only take on discrete values if living on its own, but if other matter is present the cuscaton locks on to its dynamics. An interesting exception is a cuscuton with a suitably chosen mass term, which exhibits scaling solutions even without background matter [45]. Specifically,

$$
\mathcal{L}_{0}=\mu^{2} \sqrt{|X|}-\frac{1}{2} m^{2} \phi^{2}
$$

subject to:

$$
\mu=\frac{2}{\sqrt{3}} \sqrt{m M_{P l}}
$$

leads to solutions with $\rho \propto 1 / t^{2}$ and $\phi=A / t$. These scaling solutions have a stable equation of state:

$$
w=\sqrt{\frac{8}{3}} \frac{M_{P l}}{m A} .
$$

but the model differs from the structure formation requirements in that $c_{s}=\infty$. In order to implement $c_{s} \propto \rho$ a term of form $\mathcal{L}_{1} \propto X^{n}$ with $n<1 / 2$ should be added to $K$. This doesn't affect the homogeneous solution and its equation of state at high energies, but $c_{s}$ is no longer infinite. Using Eqn. (26) we find

$$
c_{s}^{2} \propto X^{\frac{1}{2}-n} \propto \rho^{1-2 n} .
$$

so that for $n=-1 / 2$ we have $c_{s} \propto \rho$, as required for scale-invariance.

This argument may look contrived, but we repeated it here because it is "constructive" with regards to what is required from the point of view of producing fluctuations. But we now show that it can be directly motivated from the DBI action. As abundantly pointed out in the literature 29 32] this model can be associated with "stringy physics" (an association which may or may not be relevant for this paper). DBI inflation has been studied in recent papers, where the model is tweaked so as to reduce the speed of sound during inflation. However the model could also be used to implement the varying speed of sound mechanism, dispensing with inflation altogether. The DBI model for a scalar field is based on the action:

$$
\mathcal{L}=-\frac{1}{f(\phi)} \sqrt{1-2 f(\phi) X}+\frac{1}{f(\phi)}-V(\phi),
$$

where we note that we're using signature +--- (in agreement with all other literature used in this paper), as opposed to what has been used in the literature in this field. A positive $f$ has the effect of limiting the curvature and reducing the speed of sound. We choose $f=-C$ where $C$ is a positive constant, so as consider the minimal model with the opposite feature: the speed of sound increases at high energies, without any curvature capping. For $X \ll C^{-1}$ the theory has the appropriate limit $\mathcal{L} \approx X-V$, so there's no question of introducing ghosts due to the signal of $f$. Usually $X$ cannot grow above a given value $(X=1 / 2 f)$, but now it makes sense to explore the limit $X \gg C^{-1}$ for which we obtain:

$$
\mathcal{L}=\mu^{2} \sqrt{X}+\frac{\mu^{6}}{4 \sqrt{X}}-V(\phi) .
$$

which is precisely the ad-hoc $K$-essence model we constructed above (with $\mu^{2}=\sqrt{2 / C}$ ), subject to a constraint upon the coefficients of the terms in $\sqrt{X}$ and $1 / \sqrt{X}$. This finally lets us determine the value of $\rho_{\star}$, 
so important for the normalization of the spectrum, in terms of Lagrangian parameters:

$$
\rho_{\star}=\frac{3}{8} A \frac{\mu^{6}}{M_{P l}^{2}}=\frac{1}{\sqrt{2}} \frac{\mu^{4}}{w} .
$$

In the latter identity we used (29) to eliminate $A$ in terms of $w$. The normalization implied by (22) therefore requires that:

$$
\frac{(5+3 w)^{2}}{\sqrt{2} w(1+w)} \frac{\mu^{4}}{M_{P l}^{4}} \sim 10^{-10}
$$

relating field theory parameters and observables.

Is there anything wrong with choosing the opposite sign for the DBI coupling constant? As explained above, there are no ghosts in the theory, provided a corresponding change in signs is introduced elsewhere in the Lagrangian (so that the low energy limit still comes out right). But the theory certainly doesn't come from string/brane theory, since the signature of the space fixes the sign of $f$ to be positive (at least using a minimal adaptation of the argument). This is not a reason to discard it. The only problem with the theory seems to be exactly the feature we want to implement: a superluminal speed of sound. This is a more general problem with some $K$-essence models, which we now proceed to analyse.

\section{THE MAPPING BETWEEN $K$-ESSENCE AND BIMETRIC MODELS}

We now relate the two leitmotifs in this paper: bimetric VSL theories and the varying speed of sound mechanism as implemented by the DBI model. $K$-essence models can be seen as theories with an emergent second metric [20]. Although the authors of [20] distance their work from full bimetric theories, in the limit of perturbative fluctuations in a $K$-essence field the two perspectives are equivalent. So it's natural to enquire which emergent metric theory is associated with DBI models; and should we see such a metric as the signature of a bimetric theory (as we shall do in the next Section), which bimetric theory is associated with DBI models (or anti-DBI models; for the purpose of this discussion the sign of the coupling is irrelevant.)

There is a good reason why $K$-essence and DBI models are usually only invoked when they reduce, rather than increase the speed of sound: the innate fear of faster than light propagation. Causality paradoxes, such as the anti-telephone, are expected to arise (see [19, 20]; but also see [33] for an intrepid alternative). These can be circumvented by re-interpreting $K$-essence as a bimetric theory. We review this mapping deriving the remarkable result that the DBI model capable of producing scaleinvariance, as shown in Section IV] is mapped precisely into the minimal bimetric theory discussed in Section [I]
However we stress that the mapping discussed in this Section is perturbative, and an understanding of the full nonperturbative aspects of the bimetric theory will only be achieved in the next Section.

The crux of the bimetric re-interpretation discussed in [20] is the remark that the field equation of a $K$-essence theory is equivalent to a Klein-Gordon theory subject to effective metric:

$$
\widetilde{M}^{\mu \nu}=K_{, X} g^{\mu \nu}+K_{, X X} \partial^{\mu} \phi \partial^{\nu} \phi
$$

with inverse

$$
\widetilde{M}_{\mu \nu}=\frac{1}{K_{, X}}\left(g_{\mu \nu}-c_{s}^{2} \frac{K_{, X X}}{K_{, X}} \partial_{\mu} \phi . \partial_{\nu} \phi\right)
$$

This metric is generally not conformal to the metric $g_{\mu \nu}$ servicing gravity, immediately pointing us in the direction of bimetric VSL theories. As long as

$$
1+2 X \frac{K_{, X X}}{K_{, X}}>0
$$

(equivalent to requiring $c_{s}^{2}>0$ ) the system is then hyperbolic with respect to this metric and more generally the Cauchy problem is well defined, resolving causality paradoxes. So it's tempting to identify (35) with a conformal version of (11), with $\widetilde{M}_{\mu \nu}$ playing the role of matter metric $\hat{g}_{\mu \nu}$ and action (41).

We stress, however, that this mapping with bimetric theories is imperfect. Firstly it induces a field equation

$$
\widetilde{M}^{\mu \nu} \nabla_{\mu} \nabla_{\nu} \phi=0
$$

where the $\nabla$ operator is defined with respect to metric $g_{\mu \nu}$, not $\widetilde{M}_{\mu \nu}$. Secondly, a Klein-Gordon field equation for $\phi$ doesn't correspond to a Klein-Gordon Lagrangian in bimetric theories, as explained in Section III. Finally the subtleties in defining the stress-energy tensor (see Section (II) are altogether ignored. Therefore the mapping just described can only be used gingerly.

A stronger connection is established in [20], considering a background $\phi=\phi_{0}$ plus perturbations around it, a situation well suited to cosmology. Leray's theorem states that the perturbations satisfy

$$
M^{\mu \nu} D_{\mu} D_{\nu} \delta \phi=0
$$

with a new metric (conformal to $\widetilde{M}_{\mu \nu}$ ) given by

$$
M^{\mu \nu}=\frac{c_{s}}{K_{, X}}\left(g^{\mu \nu}+\frac{K_{, X X}}{K_{, X}} \nabla^{\mu} \phi \nabla^{\nu} \phi\right),
$$

where all quantities are to be defined at $\phi=\phi_{0}$. The covariant derivatives $D_{\mu \nu}$ used in defining the KleinGordon equation are now defined with respect to metric $M_{\mu \nu}$ and the theory may be derived from a Klein-Gordon Lagrangian fully built from $M_{\mu \nu}$. The mapping is therefore rigorous and we should identify the inverse metric

$$
M_{\mu \nu}=\frac{K_{, X}}{c_{s}}\left(g_{\mu \nu}-c_{s}^{2} \frac{K_{, X X}}{K_{, X}} \partial_{\mu} \phi \partial_{\nu} \phi\right)
$$


with the matter frame $\hat{g}_{\mu \nu}$ via a linearization of (1). The action for the perturbations

$$
\delta S^{(2)}=\int d^{4} x \sqrt{-M} \frac{1}{2} M^{\mu \nu} \partial_{\mu} \delta \phi \partial_{\nu} \delta \phi
$$

is then understood from a linearization of (4) or (5) via the proposed identifications (we shall ignore the potential for the time being, as only the kinetic term affects the mapping under study).

With this mapping in mind we can now ask onto what $K$-essence models one maps the minimal bimetric theory. In general any $K$-essence theory maps into a bimetric theory of form (1) but this could have a complicated form of $B=B(\phi, X)$. It is therefore interesting to ask which theories lead to a minimal bimetric theory. If we compare (1) with (41), ignoring the conformal factor, we get:

$$
-c_{s}^{2} \frac{K_{, X X}}{K_{, X}}=-\frac{K_{, X X}}{K_{, X}+2 X K_{, X X}}=B .
$$

This is equivalent to

$$
\frac{K_{, X X}}{K_{, X}}=-\frac{B}{1+2 B X}
$$

and if $B$ is constant this integrates immediately into:

$$
K=\frac{1}{B} \sqrt{1+2 B X}-\frac{1}{B}
$$

which is the DBI model (notice that the potential does not enter the expression for the speed of sound and therefore is left unconstrained).

This result has a remarkable implication. If we consider a minimal bimetric theory where the bi-scalar is Klein-Gordon in the matter frame, then in a perturbative scheme we should identify $\hat{g}_{\mu}=M_{\mu \nu}$. Then we find that in the Einstein frame, $g_{\mu \nu}$, the theory maps into a DBI model, i.e. precisely the $K$-essence model that leads to scale-invariance. Comparing with Section IV we see that we have $B=C$, so that a positive constant $B$ leads to a DBI model with $f<0$, as required. This is a perturbative result, but remarkable. It implies that scaleinvariant fluctuations follow from the minimal bimetric model, with a constant positive $B$, with a minimal action defined in the matter frame.

As a side remark notice that had we taken the original Clayton-Moffat theory, where the bi-scalar is KleinGordon in the Einstein (or VSL) frame we would have discovered that in the matter frame its dynamics maps into DBI model with $f>0$. This can be proved by a straightforward adaptation of the above calculation, but the result can be intuitively understood. In this original model the speed of sound for the bi-scalar is the speed of gravity, which in the early universe is much smaller than the speed of light. So the speed of sound for the bi-scalar in the matter frame becomes negligible, just like for a DBI model with $f>0$.

\section{THE FULL NON-PERTURBATIVE STRUCTURE}

We are now prepared to identify the full nonperturbative bimetric structure behind the various models presented in this paper. It will be simpler and more elegant, and perhaps we should have presented it upfront, bypassing the hardships of cosmology. We stress that the work in [20] is distinct from the bimetric picture to be presented in this section, and that there should be observational differences between the two, should there be further matter fields, or non-perturbative effects be under study.

Let the dynamics of bi-scalar $\phi$ be driven by a pure cosmological constant in the matter frame, that is:

$$
S_{\phi}^{1}=\int d^{4} x \sqrt{-\hat{g}}(-2 \hat{\Lambda}) .
$$

Even if we don't bestow a kinetic term upon $\phi$ (either in the matter or the Einstein frame) the field acquires dynamics, due to the subtlety explained at the end of Section [I] In the absence of $K$ and $V$ the first two terms in (11) disappear, but since $\hat{T}^{\mu \nu}=\Lambda \hat{g}^{\mu \nu}$ the field satisfies

$$
\hat{T}^{\mu \nu} \hat{\nabla}_{\mu} \hat{\nabla}_{\nu} \phi=\hat{\Lambda} \hat{g}^{\mu \nu} \hat{\nabla}_{\mu} \hat{\nabla}_{\nu} \phi=0
$$

that is, a Klein-Gordon equation in the matter frame. This is actually the only way to achieve a Klein-Gordon equation of motion in the matter frame, since a KleinGordon Lagrangian, Eqn. (4), does not translate into a Klein-Gordon equation.

In addition this theory has the required behaviour, for structure formation, to all orders in the Einstein frame. From (10) we have

$$
\hat{g}=g(1+2 B X)
$$

(see [9, 34] for a derivation) so that $S_{\phi}^{1}$ in the Einstein frame becomes

$$
S_{\phi}^{1}=\int d^{4} x \sqrt{-g} \sqrt{1+2 B X}(-2 \hat{\Lambda}) .
$$

This is already all that is needed to obtain the correct high energy behaviour and therefore scale-invariant fluctuations. If additionally we want to ensure that the theory has the correct low energy limit we must choose

$$
\hat{\Lambda}=-\frac{1}{2 B}
$$

and require that an exactly balancing cosmological constant is present in the Einstein frame. This results in the DBI action (31) with $f=-B$ (or $C=B$ ). As explained in Section IV] we still need to add a mass term in the Einstein frame, with

$$
m^{2}=\frac{3}{2 B M_{P l}^{2}}
$$


but this is in fact required by the Bianchi identities, if we want $\phi$ to remain dynamical in all circumstances.

To summarize all we need is a bimetric theory with two balancing cosmological constants, one in each frame:

$$
S_{\phi}=\int d^{4} x \sqrt{-\hat{g}} \frac{1}{B}-\int d^{4} x \sqrt{-g} \frac{1}{B},
$$

tuned to the same constant $B$ which appears in the relation between the two metrics (1). Such a theory has a Klein-Gordon dynamics in the matter frame but becomes DBI in the Einstein frame. The Bianchi identities then force it to have a mass term ensuring the appropriate background scaling solutions at high energies. Its DBI behaviour in the Einstein frame induces a varying speed of sound with the correct profile to generate scaleinvariant fluctuations with amplitude

$$
\frac{2^{3 / 2}(5+3 w)^{2}}{w(1+w)} \frac{1}{B M_{P l}^{4}} \sim 10^{-10}
$$

(see Eq. 34). Thus, with the possible exception of the equation of state $w$, nothing remains undefined in this theory.

It is possible that the reinterpretation of the anti-DBI action in the bimetric framework might shed some light on its known pathologies [41] if interpreted in a single frame (the theory only becomes anti-DBI in the gravity frame). This matter is beyond the scope of this paper.

\section{DISCUSSION}

It is often stated that units can always be defined so that the speed of light is a constant. This is true in the same way that units can always be defined so that the Hubble "constant" is indeed a constant, the acceleration of gravity is the same everywhere as it is on Earth, the Universe is not expanding, etc, etc, etc. The argument is a perfect twin and likewise the reason it makes sense to talk about varying $c$ is identical to why it makes sense to talk about the expansion of the Universe, Newton's laws, a varying $G$, etc, etc, etc: they are dimensional statements justified by the simplicity of the overall picture. For example by choosing units where the universe is not expanding all the equations of physics would become ridiculously complicated.

Nowhere is this more obvious, with regards to VSL, than in the present paper. We could always define units where $c$ is constant: this is in fact the matter frame. But then the Einstein equations become a royal mess, with the perturbation equations looking even uglier; that the speed of gravitational waves is now changing is an omen of what would then happen to the formalism. The gravitational dynamics of the theory (background and perturbations) is crying out for the VSL or Einstein frame to be used, just as it would if we recast modern cosmology into units in which the Universe isn't expanding. This is far from a technicality. For example it could be argued that the work in this paper is simply inflation 35 or ekpyrotic [36] in a different frame. This is obviously nonsense: if we erase the VSL feature from the theory by going into the matter frame Einstein's equations and all the perturbation equations are horrendously modified. This "dynamical" feature is not part of any inflation or ekpyrotic model, and no doubt the discussion of scaleinvariance would be rather different if they were to be included [23].

We have used $K$-essence models, of the DBI persuasion, as a stepping stone to the construction presented in this paper. But we believe that the bimetric VSL structure identified towards the end of the paper offers a superior description. To save $K$-essence from the perils of the causality paradoxes the theory needs to be reinterpreted as an emergent 20] or a bimetric theory. The construction obtained is then valid only perturbatively and coincides with ours to first order. But our bimetric construction is valid to all orders, and in any case has a simpler dynamics: two balancing cosmological constants, one in the matter frame the other in the Einstein frame, as discussed in Section VI.

In this paper we focused on initial conditions set by a vacuum state which is at first inside the horizon. We leave to further work establishing the connection with thermal initial conditions [1, 37, 38]. This is because these require a phase transition in the speed of light, and are best realized in a theoretical framework diametrically opposed to the one described here (see, for example, [39, 40]). The groundbreaking paper on VSL [5] provides the best set up for implementing such a phase transition. In future work [42] we shall also present the predictions made by these models concerning nonGaussianity and gravitational waves. After this paper was submitted new work appeared [43] where these issues are partly addressed. However the scope of [43] is different from our paper; notably inflation is still required.

I'd like to thank N. Afshordi, R. Brandenberger, C. Contaldi, A. Tseytlin and A. Vikman for helpful comments. I'm very grateful to John Moffat for providing the inspiration for this paper and for a careful reading of the manuscript.
[1] J. Magueijo, Phys. Rev. Lett. 100, 231302 (2008).

[2] C. Armendariz-Picon, T. Damour and V. Mukhanov, Phys. Lett. B458, 209, 1999.

[3] J. Garriga and V. Mukhanov, Phys. Lett. B458: 219-225,
1999.

[4] J. Magueijo, Rep. on Prog. in Phys. 66 (11), 2025, 2003.

[5] J. W. Moffat, Int. J. Mod. Phys. D 2, 351-366 (1993).

[6] A. Albrecht and J. Magueijo, Phys.Rev. D 59043516 
(1999)

[7] G. F. R. Ellis, astro-ph/070375.

[8] J. Magueijo and J. Moffat, arXiv:0705.4507

[9] M. A. Clayton, J. W. Moffat, Phys. Lett. B506, 177-186, 2001.

[10] I. Drummond, Phys. Rev. D63, 043503, 2001.

[11] G. Amelino-Camelia, Nature 418:34-35,2002.

[12] J. Magueijo and L. Smolin, Phys. Rev. Lett. 88: 190403, 2002; J. Magueijo and L. Smolin, Phys. Rev. D67: 044017, 2003.

[13] S. Hollands and R. Wald, Gen.Rel.Grav.34:20432055,2002

[14] C. Armendariz-Picon and E. Lim, JCAP 0312: 002, 2003; C. Armendariz-Picon JCAP 0610: 010, 2006.

[15] Y. Piao, Phys.Rev.D75: 063517, 2007.

[16] J. Magueijo, in preparation.

[17] S. Mukhanov, H. Feldman and R. Brandenberger, Phys.Rept. 215: 203-333, 1992; Physical Foundations of Cosmology, V. Mukhanov, CUP (2005).

[18] J. Lidsey et al, Rev. Mod. Phys. 69: 373-410, 1997.

[19] J. Bruneton, Phys.Rev.D75:085013,2007.

[20] E. Babichev, V. Mukhanov, A. Vikman, JHEP 0802: 101, 2008.

[21] Brans C and Dicke R H, 1961 Phys. Rev. 124925.

[22] J. Bekenstein, Phys. Rev. D70, 083509 (2004); Erratumibid. D71, 069901 (2005).

[23] M. A. Clayton, J. W. Moffat, JCAP 06 (2003) 006; M. A. Clayton, J. W. Moffat, Int.J.Mod.Phys.D12:697712,2003 .

[24] C. Bonvin, A. Caprini and R. Durrer, Phys. Rev. Lett., 97: 081303, 2006.

[25] V. Mukhanov and A. Vikman, JCAP 0602: 004, 2006

[26] R. Bean, D. Chung and G. Geshnizjani, arXiv:0801.0742,

[27] N. Afshordi, D. Chung and G. Geshnizjani, Phys. Rev.
D75: 083513, 2007.

[28] N. Afshordi et al, Phys. Rev. D75: 123509, 2007.

[29] E. Silverstein and D. Tong, Phys.Rev. D70: 103505, 2004.

[30] M. Alishhiha, E. Silverstein and D. Tong, Phys.Rev. D70: 123505, 2004.

[31] Z. Gou and N. Ohta, JCAP 0804:035, 2008.

[32] W. Kinney and K. Tzirakis, Phys. Rev.D77: 103517, 2008.

[33] E. Recami, Revista del Nouvo Cimento,9 (6), 1986.

[34] D. Langlois et al, 0806.0336; D. Langlois et al, 0804.3139.

[35] A. Guth, Phys.Rev. D23 347 (1981); A. Linde, Phys. Lett B 108, 1220 (1982)

[36] P. Steinhardt and N. Turok, Science 296: 1436-1439, 2002.

[37] P. Ferreira and J. Magueijo, arXiv:0708.0429.

[38] J. Magueijo and P. Singh, Phys.Rev.D76: 023510, 2007.

[39] A. Nayeri, R. Brandenberger and C. Vafa, Phys. Rev. Lett.97: 021302, 2006; R. Brandenberger et al, hep-th/0608121 and hep-th/0608186 Biswas et al, hep-th/0610274 R. Brandenberger et al, JCAP 0611:009,2006.

[40] J. Magueijo, L. Smolin and C. Contaldi, Class.Quant.Grav.24: 3691-3700, 2007.

[41] A. Adams et al, JHEP 0610:014, 2006.

[42] J. Noller and J. Magueijo, in preparation.

[43] J. Khoury and F. Piazza, hep-th/0811.3633.

[44] Note that the equation and third metric for (5) have a similar form.

[45] If there is background matter the cuscuton with a mass potential always scales with it. In the absence of matter by scaling we mean a solution where the density goes like $1 / t^{2}$ 